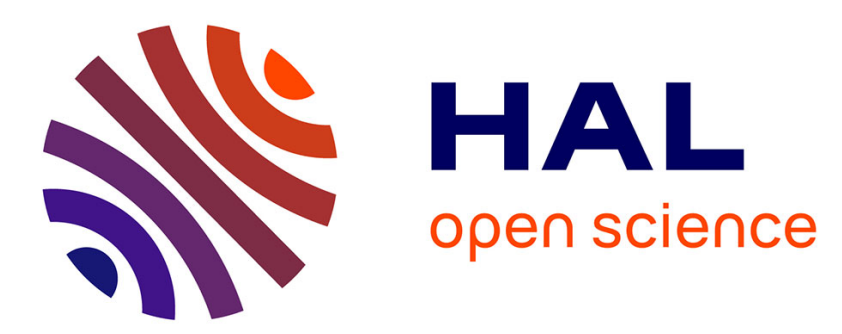

\title{
Synergistic Effects of Cardanol- and High Oleic Soybean Oil Vinyl Monomers in Miniemulsion Polymers
}

Zoriana Demchuk, W. S Jennifer Li, Hermella Eshete, Sylvain Caillol, Andriy Voronov

\section{- To cite this version:}

Zoriana Demchuk, W. S Jennifer Li, Hermella Eshete, Sylvain Caillol, Andriy Voronov. Synergistic Effects of Cardanol- and High Oleic Soybean Oil Vinyl Monomers in Miniemulsion Polymers. ACS Sustainable Chemistry \& Engineering, 2019, 7 (10), pp.9613-9621. 10.1021/acssuschemeng.9b01137 . hal-02138104

\section{HAL Id: hal-02138104 https://hal.science/hal-02138104}

Submitted on 15 May 2020

HAL is a multi-disciplinary open access archive for the deposit and dissemination of scientific research documents, whether they are published or not. The documents may come from teaching and research institutions in France or abroad, or from public or private research centers.
L'archive ouverte pluridisciplinaire HAL, est destinée au dépôt et à la diffusion de documents scientifiques de niveau recherche, publiés ou non, émanant des établissements d'enseignement et de recherche français ou étrangers, des laboratoires publics ou privés. 


\title{
Synergistic Effects of Cardanol- and High Oleic Soybean Oil Vinyl Monomers in Miniemulsion Polymers
}

Zoriana Demchuk ${ }^{1}$, W. S. Jennifer $\mathrm{Li}^{2}$, Hermella Eshete ${ }^{3}$, Sylvain Caillol ${ }^{2 *}$, Andriy Voronov $^{1 *}$

${ }^{1}$ Department of Coatings and Polymeric Materials, North Dakota State University, Fargo, ND 58105-6050

${ }^{2}$ Institut Charles Gerhardt de Montpellier CNRS, ENSCM, UM, Montpellier, France

${ }^{3}$ Department of Chemistry and Biochemistry, The University of Southern Mississippi, Hattiesburg, MS 39406

\begin{abstract}
Along with characterization of vinyl monomers from high oleic soybean oil (HOSBM) and cardanol (CBM) in free radical (co)polymerization, a series of stable latexes based on various ratios of these monomer mixtures have been synthesized using miniemulsion process. By combination of aliphatic fatty acid fragments of HOSBM with aromatic CBM structure, durable latexes from fully renewable feeds were expected, as well as the ability to balance thermomechanical properties of resulting crosslinked latex films. Biobased latexes were synthesized from 10-75 wt.\% of CBM and 25-90 wt.\% of HOSBM in the feed, characterized, and tested in films and coatings performance. Synergistic effects of both constituents in the films and coatings were observed. Incorporation of cardanol-based fragments enhances Young's modulus of the films, whereas the plant oil-based units contribute to softer and more flexible films. Even small (10 wt.\%) fractions of CBM can provide noticeable strength to the soft latex polymer material based entirely on HOSBM. All crosslinked latex films in this study exhibit decent properties and performance in terms of pendulum and pencil hardness, water and solvent resistance as well as adhesion to the steel substrate. To the best of our knowledge, latexes from fully renewable plant-based monomer mixtures have not been reported before in literature.
\end{abstract}

Keywords: plant-based monomers, renewable polymers, latexes, crosslinked latex films

\section{Introduction}


For the past few decades, the industry has been looking for new methods of synthesizing polymers and polymeric materials from renewable resources. Using renewable ingredients in the production of biobased polymers can address concerns of global lowering of the oil feedstock reserves of fossil fuels, as well as having positive environmental impact ${ }^{1}$. Additionally, polymeric materials synthesized from renewables offer a great range of application possibilities, with properties and performance which can compete and sometimes even outperform the conventional petroleum-based polymers. In this regard, extensive research studies have been performed on the synthesis and polymerization of various biobased monomers, thus providing a broad platform for the utilization of the resulting polymers in coatings, plastics, adhesives, composites, etc. ${ }^{2-5}$.

Due to its worldwide availability, low cost, abundance, rich application potentials and renewable nature, plant oils and their derivatives are widely used in the synthesis of polymers for paints, coatings, thermosetting resins, polymer composites etc. Biobased polymers from plant oil derivatives exhibit a wide range of thermo-physical and mechanical properties: from soft and flexible to hard and brittle, thus presenting a promising way of replacing petroleumbased polymers by biobased polymeric counterparts ${ }^{6,7}$. The major component in plant oils are triglycerides, esters of glycerol, and three different fatty acids possessing various amounts of unsaturations (double bond fragments), which can be modified to yield new compounds, in particular plant oil-based monomers ${ }^{8}$. In recent years, incorporation of acrylate groups and $\mathrm{OH}$-groups into plant oil molecular structure, and epoxidizing plant oil fatty acid double bonds have been successfully demonstrated ${ }^{9,10}$. Furthermore, various newly synthesized plant oil-based monomers have been investigated in the formation of polymer latex materials through emulsion and miniemulsion processes ${ }^{11,12,16}$. The incorporation of plant oil-based monomers in copolymerization with conventional petroleum-based counterparts provides several benefits to the final copolymers such as plasticizing properties, the elimination of coalescence aids in latex applications, reducing minimum film formation temperature and film-forming properties. The ability of fatty acid fragments to undergo autoxidation (due to presence of allylic double bonds in resulting macromolecules) after latex application and yield the crosslinked polymer networks can balance the mechanical properties of final latex products, which can be beneficial as well ${ }^{13,17,20}$.

Another interesting plant-based renewable resource which has attracted a lot of attention in the last few years is cardanol. This is a by-product from the cashew nut industry and refers to the aromatic class of organic compounds. Cardanol is extracted from cashew nut shell liquid after its thermal treatment. The combination of a benzene ring and long fatty acid 
chains in its structure provides a lot of opportunities in terms of tuning material performance $^{14}$. Due to the fascinating chemical structure, cardanol has multiple reaction sites, which can be used for further functionalization. Both cardanol and cardanol derivatives have been applied in synthesis of materials for different applications, such as surfactants, plastics, thermosets, plasticizers, antioxidants, etc. ${ }^{18-21}$. Cardanol was reported to be used in the synthesis of cardanol-based benzoxazine resin for biocomposite formation ${ }^{22}$. Also, cardanol methacrylates were applied in polymer synthesis for dental applications and provided a noticeable reduction of dentin permeability and resistance to acid exposure of the resulting materials $^{23}$.

We hypothesized that synthesis of emulsion polymers from cardanol- and plant oil-based monomers might provide an opportunity to replace petroleum-based constituents in latex synthesis, as well as to reduce VOC content in resulting polymeric materials. However, due to the highly hydrophobic nature of both natural components, synthesis of latexes using conventional emulsion polymerization can be challenging. Recently, we reported on emulsion polymers with biobased content of up to $60 \%$ wt. synthesized in miniemulsion from new vinyl monomers derived from a variety of plant oils using transesterification reaction ${ }^{15-17}$. Incorporation of plant oil-based fragments into the latex copolymers provided plasticization and hydrophobization, as well as improved toughness of the final crosslinked latex polymer films. Nevertheless, by increasing plant oil-based content in the latex copolymers above 60 wt.\%, excessive softening of polymeric materials has been experienced to the point where it becomes "too soft" and not applicable for latex film preparation. To be able to further increase the biobased content in the emulsion polymers without deteriorating the material properties and performance, cardanol methacrylate (CBM) was considered as a comonomer in the miniemulsion process with high oleic soybean oil-based (HOSBM) monomer. CBM was synthesized in a two-step reaction by cardanol hydroxyethylation and further methacrylation $^{24}$, as well as copolymerized with methyl methacrylate to form latexes in miniemulsion $^{25}$.

To benefit from HOSBM and CBM chemical structures and their ability to undergo radical polymerization, this study combines both newly synthesized biobased monomers in miniemulsion to yield latex copolymers with various ratios of CBM/HOSBM. It is expected that this fully renewable monomer feed yields a range of new emulsion polymers with properties and performance enhanced by plasticising HOSBM residues and strengthimproving CBM fragments, acting synergistically. Additionally, the presence of fatty acid unsaturated fragments in both monomers provide an opportunity to crosslink latex films to 
different degrees and, thus, control the resulting polymer network mechanical properties. To the best of our knowledge, latexes from fully renewable plant-based monomer mixtures have not been reported before in literature.

\section{Materials and Methods}

Materials. High oleic soybean oil (Perdue Agribusiness LLC, Salisbury, MD), N(hydroxyethyl) acrylamide (TCI America, Portland, OR), cardanol NX2026 (Cardolite), ethylene carbonate (99\%, Aldrich), 1,5-diazabicyclo[4.3.0]non-5-ene (DBN, 98\%, Aldrich), triethylamine (99.5\%, Aldrich), methacrylic anhydride (94\%, Aldrich), sodium hydroxide (98\%, Alfa Aesar, Ward Hill, MA), sodium sulphate (99\%, Aldrich) sodium dodecyl sulphate (VWR, Radnor, PA), sodium chloride (ACS grade, VWR, Radnor, PA) were used as received. 2,2'-Azobis(2-methylpropionitrile) (AIBN; Sigma-Aldrich, St. Louis, MO) was purified with recrystallization from methanol. All solvents (tetrahydrofuran, dichloromethane, methanol) were reagent grade or better and were used as received. Deionized water was used for all reactions (Milli-Q, $18 \mathrm{M} \Omega$ ).

Synthesis of HOSBM. High oleic soybean oil-based monomer (HOSBM) was synthesised via one-step transesterification reaction of crude high oleic soybean oil with N-hydroxyethyl acrylamide (HEAA) in the presence of $1.5 \mathrm{wt} \%$ of sodium hydroxide $(\mathrm{NaOH})$ as catalyst. The resulting monomer mixture was purified using $5 \mathrm{wt}$. \% brine solution, dried under a magnesium sulphate layer with continuous stirring and rotary evaporated to remove the solvent. Detailed procedure of the plant oil-based monomer synthesis can be found in ${ }^{26}$.

Synthesis of CBM. Cardanol methacrylate (CBM) was synthesized using the facile one-pot two-step reaction through hydroxyethylation of the phenolic hydroxyl group of pure cardanol, followed by a methacrylation step of the cardanol derivative. Detailed description of CBM synthesis and characterization can be found $\mathrm{in}^{24}$.

Characterization of HOSBM and CBM. Synthesized HOSBM and CBM were characterized using ${ }^{1} \mathrm{H}$ NMR spectroscopy (AVANCE III HDTM 400 high performance digital NMR spectrometer, BRUKER, Billerica, MA) using d-dichloromethane as a solvent to confirm chemical structure of the monomers.

Iodine value. Using a standard procedure, iodine value (IV) was determined in order to characterize biobased monomers in terms of the amount of unsaturation. $0.1 \mathrm{~g}$ of each monomer sample was placed in a $500 \mathrm{~mL}$ glass flask and dissolved in $20 \mathrm{ml}$ of $\mathrm{CCl}_{4} .25 \mathrm{ml}$ of Wijs solution was added to the mixture and kept in a dark place for $1 \mathrm{~h}$ at $20^{\circ} \mathrm{C}$. After the incubation period, $30 \mathrm{~mL}$ of $0.1 \mathrm{~N} \mathrm{KI}$ solution was mixed with $100 \mathrm{~mL}$ of cold boiled water 
and added to the flask. The resulting solution was titrated with $0.1 \mathrm{~N}$ sodium thiosulfate solution with constant mixing. The addition of $\mathrm{Na}_{2} \mathrm{~S}_{2} \mathrm{O}_{3}$ was continued until the yellow colour had almost disappeared; $3 \mathrm{ml}$ of starch indicator solution was added to the flask, and the titration continued until the solution became white. A blank test was conducted at the same time.

Iodine value was calculated as grams of iodine absorbed per $100 \mathrm{~g}$ sample using the following formula:

$$
I V=\frac{\left(V_{B}-V_{S}\right) \cdot N \cdot 12.69}{m_{S}}
$$

where $V_{B}$ and $V_{S}$ are volumes of $\mathrm{Na}_{2} \mathrm{~S}_{2} \mathrm{O}_{3}$ solution used for the titration of the blank and monomer samples, respectively; $N$ - normality of $\mathrm{Na}_{2} \mathrm{~S}_{2} \mathrm{O}_{3}$ solution; $m_{s}-$ mass of the monomer sample used for titration.

Free Radical (Co)Polymerization of CBM and HOSBM in Solution. For homopolymerization, HOSBM and/or CBM and AIBN were dissolved in toluene. The reaction mixture was purged with nitrogen at room temperature and heated at $75{ }^{\circ} \mathrm{C}$ for $5 \mathrm{~h}$ under continuous stirring.

To determine the reactivity of CBM and HOSBM in copolymerization, biobased monomers were copolymerized with styrene $(\mathrm{St})$ at various ratios ([CBM or HOSBM]: St $15: 85,30: 70,45: 55,55: 45,70: 30$ and $85: 15$, wt. $\%$ ) in toluene at $75^{\circ} \mathrm{C}$ under a nitrogen blanket for $3 \mathrm{~h}$ until total monomer conversion of $10-15 \%$ was reached. Copolymer samples $(0.5 \mathrm{~g})$ were taken out from the flask to check the extent of the copolymerization using gravimetry after precipitation of the copolymers in methanol and drying under nitrogen flow.

Polymers synthesized in solution were precipitated in a large excess of methanol and purified three times by reprecipitation. The final (co)polymer samples were dried under nitrogen flow until constant mass. The average number and weight molecular weight of the (co)polymers were determined by gel permeation chromatography (GPC) and their chemical structure and composition was characterized by ${ }^{1} \mathrm{H}$ NMR spectroscopy.

Biobased latex synthesis. A series of biobased latexes were synthesised using miniemulsion polymerization of CBM and HOSBM at various ratios. To synthesize latexes with $30 \%$ solids content, the oil phase was prepared by mixing certain amounts of HOSBM (3.75-13.5 g) and CBM (1.5-11.25 g) and 0.23 g of AIBN (1.5 wt.\% of oil phase) as initiator.

The aqueous phase was prepared by dissolving $0.6 \mathrm{~g}$ of surfactant (SDS, $4 \mathrm{wt} . \%$ ) and 0.04 $\mathrm{g}$ of sodium chloride $(0.02 \mathrm{M})$ in distilled water under constant stirring. The pre-emulsion formation started by drop-wise addition of the oil phase to the aqueous phase at a high 
stirring rate (800 r.p.m) and mixing for $40 \mathrm{~min}$. In order to form the miniemulsion, the preemulsion was placed in an ice bath and sonicated using a Q-Sonica digital sonicator $(500 \mathrm{~W}$, 1-inch tip, 20kHz, Newtown, CT) for 4 minutes with high energy pulses of 1 min each.

The resulting stable miniemulsions were placed in a round-bottom flask, purged with argon for 5 minutes and polymerized at $75{ }^{\circ} \mathrm{C}$ for 6 hours under continuous stirring at 350 r.p.m.

Characterization of biobased latexes and latex films. The latex polymer yield was measured by gravimetry. For this purpose, latex aliquots were reprecipitated in methanol three times to remove all unreacted monomer residuals. The precipitated polymer samples were dried under nitrogen flow to constant mass. The latex solids content was determined by drying the latex sample on an aluminium dish in the oven at $120^{\circ} \mathrm{C}$ for $45 \mathrm{~min}$.

The latex copolymers composition was examined using ${ }^{1} \mathrm{H}$ NMR spectroscopy after dissolving the biobased copolymers in $\mathrm{CDCl}_{3}$.

Number and weight average molecular weight of latex copolymers was determined in gel permeation chromatography (GPC) using a Waters Corporation modular chromatograph which consists of a Waters 515 HPLC pump, a Waters 2410 Refractive Index Detector, and a set of two $10 \mu \mathrm{m}$ PL-gel mixed-B columns; the column temperature was set at $40{ }^{\circ} \mathrm{C}$ and THF was applied as a carrier with $0.35 \mathrm{ml} / \mathrm{min}$ of flow rate.

Latex particles sizes and size distributions were determined by dynamic light scattering (DLS) (Particle Sizing Systems Nicomp 380, Santa Barbara, CA). For DLS measurements, latex solutions were prepared by adding 1 drop of latex to $5 \mathrm{ml}$ of Millipore water. The prepared samples were analysed at a $90^{\circ}$ scattering angle and the average of five measurements was calculated.

The glass transition temperature $\left(T_{g}\right)$ of latex copolymers was determined using differential scanning calorimetry (DSC) (TA instruments Q 1000 calorimeter) with a heating/cooling/heating regime in the temperature range of -50 to $150{ }^{\circ} \mathrm{C}$ with a dry nitrogen purge of the sample at $50 \mathrm{ml} / \mathrm{min}$. The latex polymer samples $(10-20 \mathrm{mg})$ were heated $/$ cooled at a rate of $10^{\circ} \mathrm{C} / \mathrm{min}$.

Biobased latex coatings and free films were prepared by applying $5 \mathrm{ml}$ of latex sample, using a drawdown bar with a thickness of $8 \mu \mathrm{m}$, on steel panels (for coatings) or glass substrates (for preparing free films) and cured at $135{ }^{\circ} \mathrm{C}$ for $4-5$ hours in the oven using autoxidation mechanism without the addition of catalyst. The resulting biobased films were peeled off from the glass substrate. The thickness of the latex coatings and free films was measured using a Byko-test 8500 coatings thickness gauge. 
Crosslinked latex films were characterized using dynamical mechanical analysis (DMA) (TA Instruments Q 800) in the temperature range of -50 to $150{ }^{\circ} \mathrm{C}$ with a heating rate of 5 ${ }^{\circ} \mathrm{C} / \mathrm{min}$ and a soaking time of $3 \mathrm{~min}$ at $-50{ }^{\circ} \mathrm{C}$. Rectangular-shaped latex films were analysed in order to evaluate its dynamical mechanical behaviour. Then, the crosslink density of the latex free films $(v)$ was determined using rubber elasticity theory:

$$
v=\frac{G \cdot \cdot 1000}{R \cdot T}, \mathrm{mmol} / \mathrm{cm}^{3}(\mathbf{2})
$$

where $G^{\prime}$ - storage modulus within the rubbery plateau; $R$-gas constant; $T$-absolute temperature.

$T_{g}$ of crosslinked latex films was taken as the temperature of $\tan \delta=f(\mathrm{~T})$ peak maximum ${ }^{27}$.

Mechanical behaviour of biobased latex free films was evaluated using an Instron tensile testing machine (Model 2710-004) with maximum load of $500 \mathrm{~N}$. The tested films had a rectangular shape with a constant width of $5 \mathrm{~mm}$. The dimensions of latex free films were evaluated before each measurement.

Biobased latex coatings performance was characterized by measuring the hardness, flexibility, water/solvent resistivity, and adhesion of each selected coating to the metal substrate. The hardness of the latex coatings was measured using pencil and pendulum hardness tests according to ASTM D3363-05 and ASTM D4366-16 standards respectively. Latex coating flexibility was evaluated using impact test (ASTM D2794) which demonstrates the ability of tested coating to "absorb" an attack from external impact without forming cracks on the surface, and conical mandrel bend test (ASTM D522) which shows bending behaviour. The solvent and water resistivity were estimated using the water/solvent double rubs test according to ASTM D4752, which counts the number of rubs applied to dissolve the surface of the coating. The adhesion of the latex coating to the metal substrate was characterized using ASTM method 3359 by applying and peeling pressure-sensitive tape over the cross-cut made on the coating surface.

To evaluate the extent of biorenewability of the synthesized latexes, the biorenewable carbon index (BCI) was calculated for all copolymers in this study using the established method $^{32}$. BCI expresses the ratio of carbon atoms which comes from renewable resources to the total amount of carbon atoms present in the molecule and can be calculated using the following equation:

$$
B C I=\frac{N_{\text {biorenew. }}^{C}}{N_{\text {total }}^{C}} \cdot 100 \%,(\mathbf{3})
$$


where $N_{\text {biorenew. }}^{C}$ - is the number of carbon atoms from renewable resources in the molecule; $N_{\text {total }}^{C}$ is the total number of carbon atoms in the molecule.

\section{Results and Discussions}

Successful syntheses of CBM and HOSBM have been reported in our recent publications along with both monomers' feasibility in free radical (co)polymerization, including emulsion and miniemulsion processes ${ }^{15-20}$. For the previously reported plant oil-based monomers, the rate of polymerization depends noticeably on their chemical structure and is higher for the monomers derived from lower quantities of unsaturated plant oil. This retardation effect (causing lower final molecular weight of polymers) is due to allylic inhibition, which is a chain transfer reaction to the fatty acid double bonds resulting in chain growth coexisting with the transfer reactions during polymerization.

With this finding in mind, in order to evaluate the extent of unsaturation in HOSBM and $\mathrm{CBM}$, the iodine value was determined for both monomers. The iodine value was noticeably higher for CBM (IV=202) compared to HOSBM (IV=123) due to natural differences in

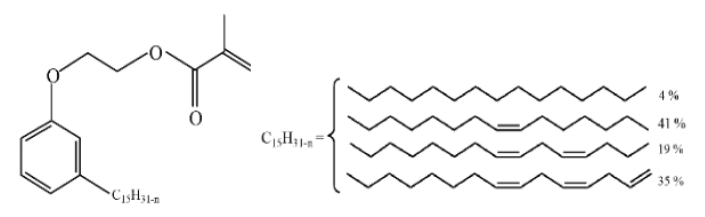

A

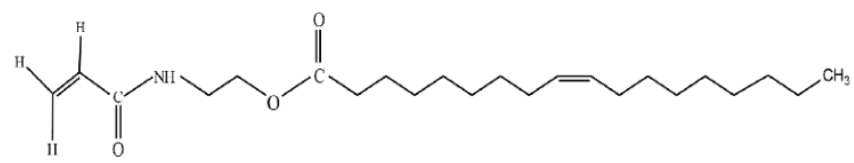

B

unsaturations in parent cardanol and high oleic soybean oil.

Figure 1. Chemical structure of CBM (A) and HOSBM (B).

To establish the effects of CBM and HOSBM unsaturation amounts on the reaction rate, homopolymerization of both monomers was carried out in solution. It is well known that radical polymerization rate is described by equation (4), showing a first order dependence of $R_{\mathrm{p}}$ on the monomer concentration $([\mathrm{M}])$ and square root dependence on the initiator concentration $([\mathrm{I}])^{15}$ :

$$
R_{p}=-\frac{d[M]}{d t}=k_{p} \cdot\left(\frac{k_{d} f[I]}{k_{t}}\right)^{\frac{1}{2}} \cdot[M],(\mathbf{4})
$$

where $f$ is the initiator efficiency, $k_{\mathrm{d}}, k_{\mathrm{p}}, k_{\mathrm{t}}$ are the rate constants of initiator decomposition, propagation and termination, respectively. 
HOSBM and CBM were homopolymerized in toluene at four different $[\mathrm{M}](0.25-1.5 \mathrm{M})$ and $[\mathrm{I}](0.02-0.06 \mathrm{M})$ to examine kinetic features and determine monomer and initiator reaction orders. Polymerization of HOSBM and CBM was carried out to monomer conversions lower than $15 \%$, in order to keep [M] constant throughout the kinetic period for calculating the polymerization rate. Figure $\mathbf{2 A}$ shows the dependence of the calculated polymerization rate $R_{p}$ on $[\mathrm{M}]$ for HOSBM and $\mathrm{CBM}$ and clearly indicates that $\mathrm{W}$ is proportional to the first power of concentration for each monomer (order of reaction with respect to monomer is 1$)$.

To further investigate the kinetics of HOSBM and CBM homopolymerization, the reaction rate and order with respect to the initiator were determined. Figure $\mathbf{2 B}$ shows the dependence of $\mathrm{W}$ on $[\mathrm{I}]$ and indicates a reaction order of $1-1.37$, deviating from normal kinetics of the polymerization of vinyl monomers.

The initiator order is higher for the polymerization of more unsaturated CBM, which can be expected based on degradative chain transfer reactions established for plant oil-based monomers in our previous publications and the fact that the calculated IV is higher for CBM in comparison to HOSBM. The latter observation is in good agreement with number average molecular weight $\left(M_{n}\right)$ values for both homopolymers, which is $26,000 \mathrm{~g} / \mathrm{mol}$ for poly(HOSBM) and 14,000 g/mol for poly(CBM).
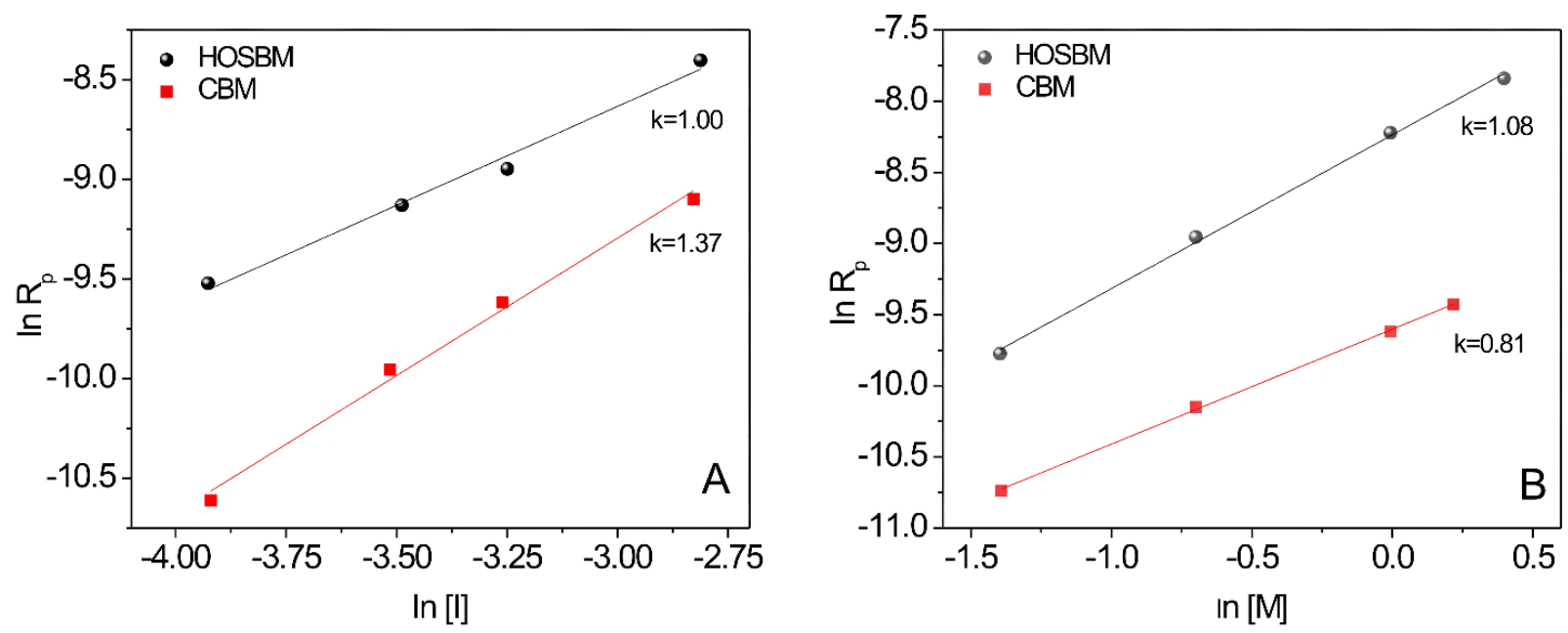

Figure 2. Rate of polymerization vs. initiator concentration (A) and monomer concentration (B) for HOSBM and CBM.

To further evaluate the extent of the effect of chain transfer on polymerization of HOSBM and CBM, the Mayo method was employed ${ }^{20}$ to determine the values of $C_{M}$ (ratio of the 
chain transfer and propagation rate constants) in the polymerization of biobased monomers at $75^{\circ} \mathrm{C}$. In this method, the inverse value of the number-average degree of polymerization is plotted against the polymerization rate, and $C_{M}$ is determined by the intercept or by extrapolating to a rate of zero.

As expected, the obtained $C_{M}$ depends on the monomer structure (Figure 3). This value is significantly higher in the case of more unsaturated CBM. It is seen from the obtained results that during $\mathrm{CBM}$ and HOSBM polymerization, chain propagation coexists with effective chain transfer to monomer (due to the abstraction of allylic hydrogen in fatty acids fragments and the fact that new macroradicals do not readily propagate the chains), and this effect is more pronounced for more unsaturated CBM.

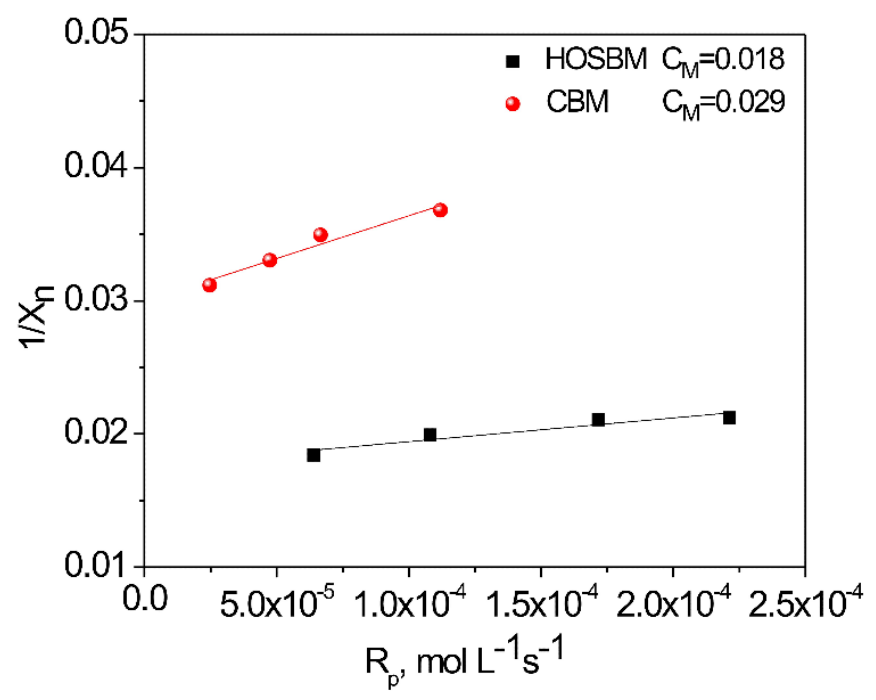

Figure 3. Monomer chain transfer constant $\left(\mathrm{C}_{M}\right)$ in the polymerization of HOSBM and CBM determined using the Mayo method.

Our previous study has shown that the fraction of chains taking part in chain transfer of allylic atoms, in fact, is not very high (8-12\%) and that it does not vary significantly among the synthesized plant oil-based monomers ${ }^{15}$. Both CBM and HOSBM are expected to behave likewise. The number-average molecular weights of poly(HOSBM) and poly(CBM) were determined to be $18,500-21,000 \mathrm{~g} / \mathrm{mol}$ and $11,000-13,000 \mathrm{~g} / \mathrm{mol}$ respectively, confirming effects of higher amount of unsaturation in cardanol-based monomer.

To describe HOSBM and CBM behaviour in chain copolymerization, Q-e parameters, which provide a semi-quantitative prediction for monomer reactivity ratios, were experimentally determined. For this purpose, instantaneous copolymer compositions in the copolymerization of HOSBM and CBM with styrene (at total monomer conversions below $10 \%$, determined by ${ }^{1} \mathrm{H}$ NMR spectroscopy) were measured. Furthermore, employing the 
Fineman-Ross method (to determine monomer reactivity ratios $r_{1}$ and $r_{2}$ ) and Alfrey-Price scheme, quantitative descriptors $Q-e$ were calculated for HOSBM and CBM.

The obtained results $(\mathrm{Q}=0.44$ (HOSBM), 0.54(CBM); e=0.14 (HOSBM), 0.51(CBM)) showed similarity, as it was expected, assuming the similarities in the chemical structures of the monomer molecules. The determined $Q$ - $e$ values can be used to estimate HOSBM and CBM reactivity in copolymerization with other monomers in order to control biobased content in the final copolymer compositions.

With a confirmed feasibility of both plant-based vinyl monomers in radical polymerization, latexes from HOSBM, CBM and their mixtures were synthesized using a miniemulsion process. For this purpose, the oil phase from various mixtures and radical initiator (AIBN) were miniemulsified in a surfactant-containing aqueous phase. Polymerization started by heating the miniemulsions at $75^{\circ} \mathrm{C}$ under stirring and was completed within 3 hours, during which most of the monomers were polymerized; the conversions of the monomers were $75-85 \%$.

Resulting latexes with an average particle size of 50-130 nm and containing 30 wt. \% solids (biobased polymer) were stable at room temperature for several months (Table 1). The molecular weights of the resulting copolymers decreased with an increasing content of unsaturated CBM in the feed, corresponding to more chain transfer reactions, as discussed above. Nevertheless, the more pronounced chain breaking reactions did not impact the total monomer conversion to the extent as that observed for HOSBM-based reactions. Table 1 shows that the mixtures with higher $\mathrm{CBM}$ concentration polymerized to overall higher monomer conversions. One plausible explanation for this observation can be the differences in the reactivities of CBM ("methacrylate") and HOSBM ("acrylate") radicals in chain copolymerization.

One additional benefit of using CBM in latex copolymerization of highly hydrophobic plant-based monomers is that this liquid substance at room temperature can be used as a (reactive) diluent for significantly more waxy HOSBM counterparts. This facilitates a much more consistent preparation of the monomer feed and, thus, the miniemulsion.

Table 1. Characteristics of CBM and HOSBM latexes synthesized in miniemulsion.

\begin{tabular}{ccccccc}
\hline \multicolumn{2}{c}{$\begin{array}{c}\text { Composition in } \\
\text { feed, \%wt. }\end{array}$} & \multicolumn{2}{c}{$\begin{array}{c}\text { Composition in } \\
\text { polymer, \% wt. }\end{array}$} & S, \% & $\begin{array}{c}\text { Solid content, } \\
\text { \% }\end{array}$ & $\begin{array}{c}\text { Mn, } \\
\text { g/mol }\end{array}$ \\
\hline CBM & HOSBM & CBM & HOSBM & & & \\
100 & 0 & 100 & 0 & 84 & 29 & 14,500 \\
75 & 25 & 85.8 & 14.2 & 81 & 31 & 18,400 \\
50 & 50 & 44.5 & 55.5 & 72 & 29 & 19,000 \\
\hline
\end{tabular}




\begin{tabular}{ccccccc}
\hline 25 & 75 & 25.5 & 76.5 & 70 & 29 & 19,300 \\
10 & 90 & 12.9 & 87.1 & 78 & 29 & 24,920 \\
0 & 100 & 0 & 100 & 71 & 20 & 26,000 \\
\hline
\end{tabular}

As determined by ${ }^{1} \mathrm{H}$ NMR spectroscopy (Figure 4), chemical compositions of the resulting copolymers mainly followed the composition of the initial monomer feed (Table 1). The polymerization yielded latexes with $100 \mathrm{wt} \%$ plant-based polymer content. As the NMR spectrum shows, a vast majority of double bonds in HOSBM and CBM fatty fragments were retained after polymerization. This indicates that fatty acid double bonds are available for post-polymerization reactions, particularly for cross-linking of latex films and polymer network formation. These findings are in good agreement with our previous results ${ }^{15-17}$.

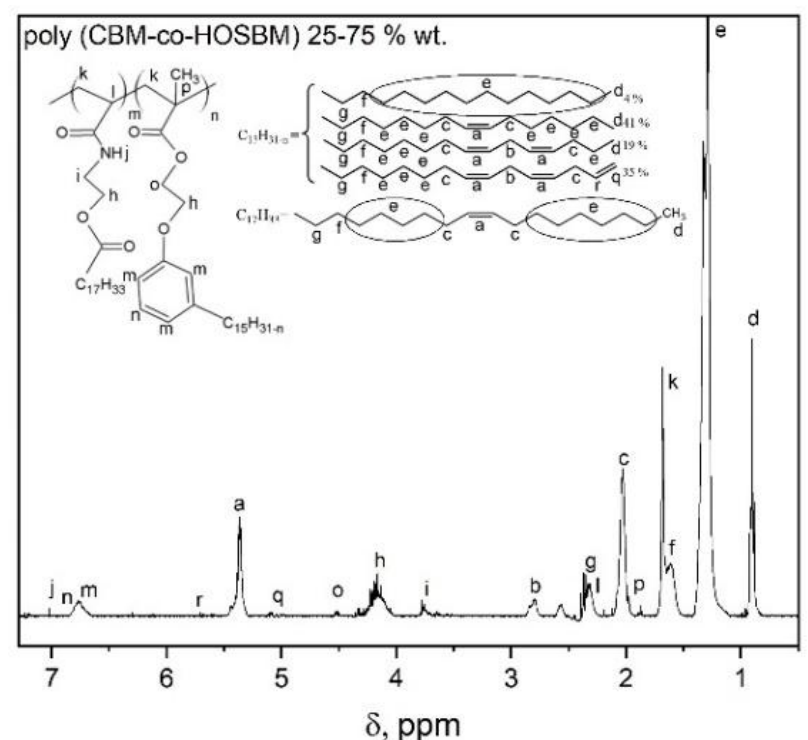

Figure. 4. ${ }^{1} \mathrm{H}$ NMR spectrum of poly (CBM-co-HOSBM) latex copolymer
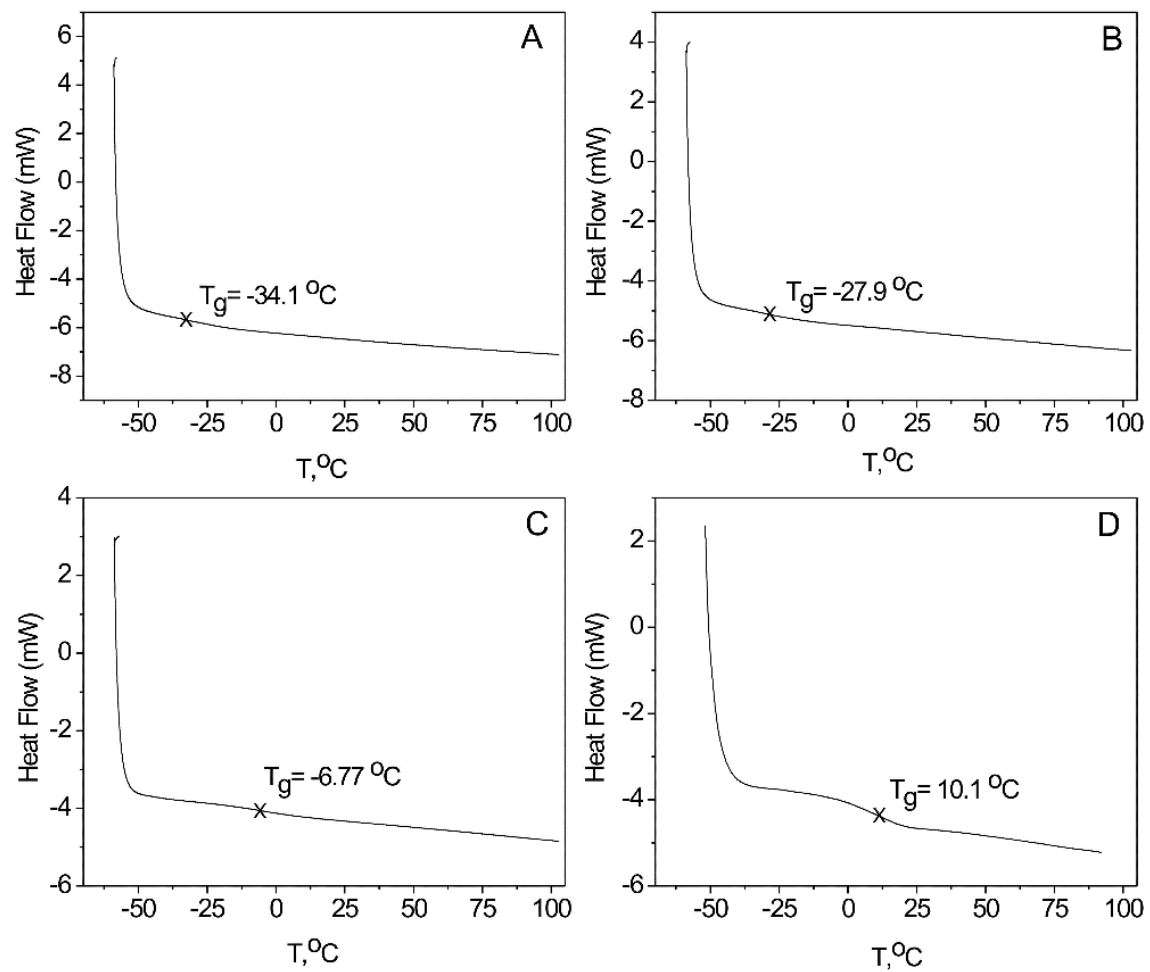
Figure 5. DSC thermograms of latex polymers from 100\% CBM (A), 75\% CBM and $25 \%$ HOSBM (B) $50 \%-50 \%$ (C) 100\% HOSBM (D) in the feed.

Glass transition temperature $\left(T_{g}\right)$ is one of the most important thermophysical properties of amorphous polymers, which gives an indication of their suitability for specific applications. The determination of $T_{g}$ by DSC of latex polymers from CBM and HOSBM showed that the synthesized biobased copolymers all possess a $T_{g}$ in the lower temperature region (-35 $10^{\circ} \mathrm{C}$ ), and that this value increases when HOSBM content in the copolymer increases (Figure 5). The obtained values indicate that variation in the biobased content in the macromolecules changes the thermal properties of latex copolymers to a large extent.

The presence of fatty acid unsaturations in HOSBM and CBM fragments makes the resulting latex polymers suitable for post-polymerization crosslinking, which can improve thermomechanical properties of the materials due to polymer network formation. Hardness, tensile strength, and Young's modulus all depend on the crosslinked polymer network structure, particularly crosslink density, $v$. The mobility of polymer chains in crosslinked networks is a function of $v$ and thus, determines the mechanical and thermal properties of a material.

In this work, biobased latex free films and coatings were formed by applying latexes onto steel substrates and curing them at an elevated temperature for 4 hours to crosslink via autoxidation mechanism, without catalyst (dryer). The resulting films were characterized using dynamic mechanical analysis (DMA) in order to determine the crosslink density, glass transition temperature and storage modulus, $E^{\prime}$. After curing, the films showed an extent of crosslinking of $75-82 \%$, as determined by gel content measurements for each latex sample.
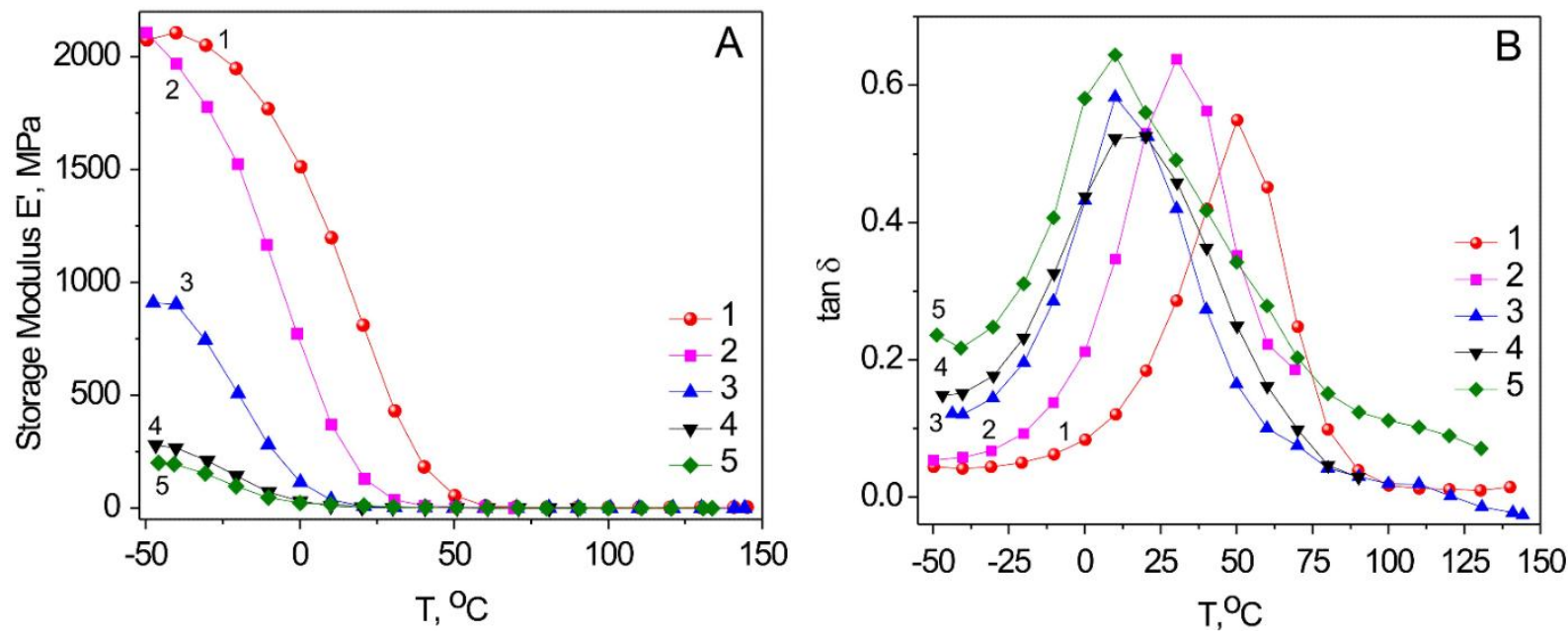
Figure 6. Storage modulus ( $\left.E^{\prime}\right)(\mathbf{A}) v s$. temperature and DMA tan $\delta$ plots (B) of biobased crosslinked latex free films from 100\% CBM - 1, 75\% CBM and 25\% HOSBM - 2, 50\% $\mathrm{CBM}$ and $50 \% \mathrm{HOSBM}-3,25 \% \mathrm{CBM}$ and $75 \% \mathrm{HOSBM}-4,10 \% \mathrm{CBM}$ and $90 \%$ HOSBM -5 .

Crosslink density, $v$, of the polymer networks was calculated using Eq. 2 where the value of the storage modulus was determined from the $E^{\prime} v s$. T dependence in the rubber elasticity region $\left(50^{\circ} \mathrm{C}\right.$ above $T_{g}$ ) (Figure $6 \mathrm{~A}$ ). The increasing $\mathrm{CBM}$ content in the monomer feed increases its unsaturation content, which results in an increased crosslink density of the polymer network, as the obtained results indicate (Table 2).

The latter observation is in good agreement with the increasing glass transition temperature values obtained for the crosslinked latex free films (Table 2) when the unsaturation content of the feed increases. The mobility of polymer chains decreases in higher CBM-based networks, thus impacting $T_{g}$.

Table 2 Cured Biobased Latex Films Properties

\begin{tabular}{cccc}
\hline $\begin{array}{l}\text { Composition in feed, \%wt. } \\
\text { CBM }\end{array}$ & HOSBM & Crosslink density $\mathbf{1 0} \mathbf{5}^{\mathbf{5}} \mathbf{\mathbf { m o l } / \mathbf { c m } ^ { \mathbf { 3 } }}$ & $\mathbf{T}_{\mathbf{g}},{ }^{\circ} \mathbf{C}$ \\
\hline 10 & 90 & 26.1 & 5.2 \\
25 & 75 & 31.4 & 13.7 \\
50 & 50 & 54.6 & 15.5 \\
75 & 25 & 115.4 & 31.2 \\
100 & 0 & 137.3 & 53.0 \\
\hline
\end{tabular}
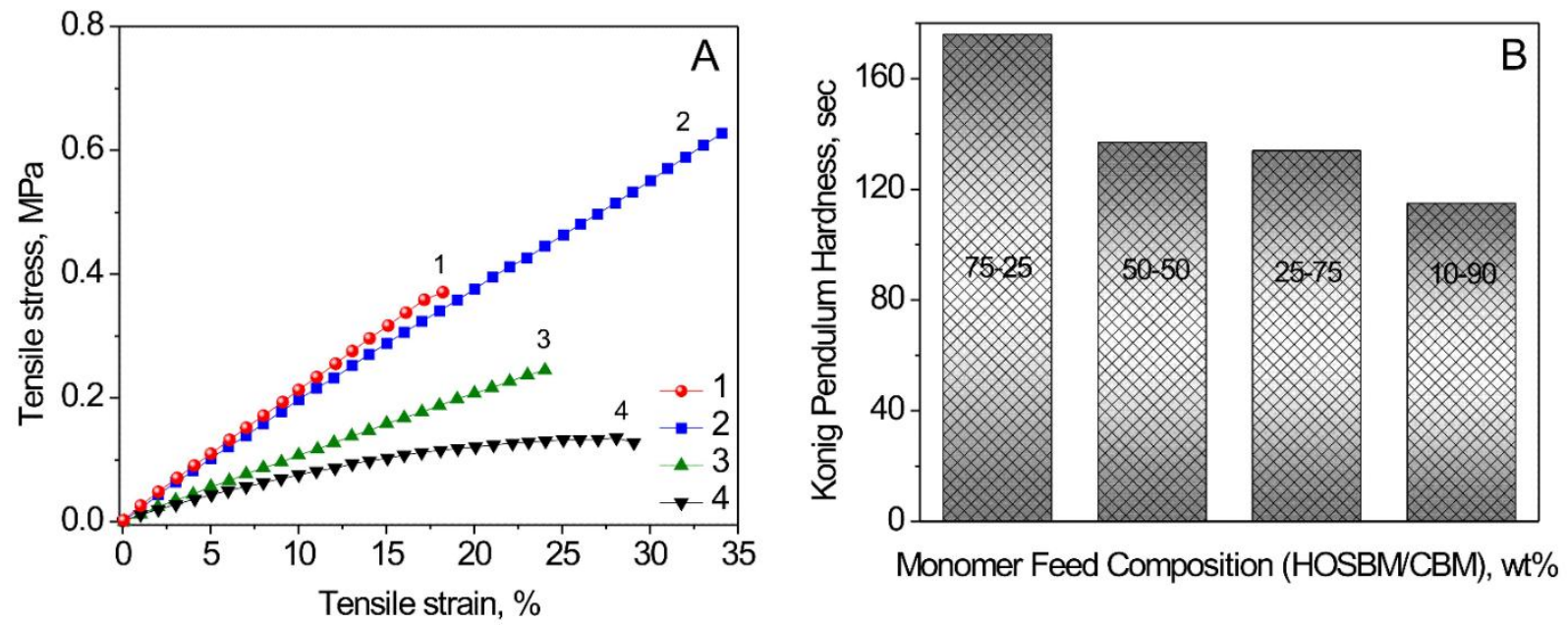
Figure 7. Stress-strain plots of biobased latex films from 100\% CBM - 1, 50\% CBM and $50 \%$ HOSBM - 2, 25\% CBM and 75\% HOSBM - 3, $10 \% \mathrm{CBM}$ and $90 \%$ HOSBM - 4 (A). Effect of HOSBM-CBM copolymer composition on pendulum hardness of biobased latex coatings $(\mathbf{B})$.

It is worth pointing out that absolute values of the storage modulus $E^{\prime}$ 'determined using DMA (Figure 6A) justify the trend observed for $T_{g}$ which can be explained by the higher crosslink density of films from mixtures with higher CBM content. Respectively, more flexible films were developed from copolymers with higher contents of less unsaturated HOSBM.

To provide more insight on the effect of different plant-based fragments on the thermomechanical properties of latex films, tensile properties were evaluated at room temperature as a function of biobased content. Toughness, Young's modulus, and elongation at break were determined for each film (Figure 7A and Table 3).

While Young's modulus and toughness increase with an increasing CBM content, both plant-based constituents contribute to the tensile properties of the latex films. The presence of HOSBM fragments makes the films more flexible, as the data demonstrates.

\begin{tabular}{cccc}
\hline $\begin{array}{c}\text { Composition in feed, \% wt. } \\
\text { CBM }\end{array}$ & HOSBM & Young's modulus, MPa & $\begin{array}{c}\text { Toughness· } \mathbf{1 0}^{\mathbf{4}}, \\
\mathbf{M J} / \mathbf{m}^{\mathbf{3}}\end{array}$ \\
\hline 10 & 90 & 0.9 & 7.0 \\
25 & 75 & 1.1 & 5.5 \\
50 & 50 & 1.9 & 13.6 \\
100 & 0 & 2.3 & 15.0 \\
\hline
\end{tabular}

Table 3. Cured biobased latex films properties

Therefore, mechanical properties of biobased latex films depend considerably on the crosslinking density of the polymer network, which can be controlled by the presence and ratio of HOSBM and CBM. The incorporation of cardanol-based fragments enhanced the Young's modulus of the films, whereas the high oleic soybean oil-based constituents made them softer and more flexible. To this end, even $10 \%$ of CBM provided noticeable strength to the entirely weak poly(HOSBM) latex polymer material. 
In addition to tensile properties, plant-based fragments can impact water and solvent resistance, hardness and adhesion of biobased latex coatings. Using ASTM methods, Konig pendulum and pencil hardness of biobased latex coatings were evaluated (Figure 7B and Table 4). As expected, the hardness was higher for coatings formulated with more unsaturated latexes containing higher CBM content, thus giving greater crosslink density networks (Figure 7B).

Next, flexibility and bending behaviour of latex copolymer coatings were characterized. The impact and conical mandrel bend test showed the highest values, which indicate excellent flexibility and bending behavior of the tested materials (Table 4). This superior behavior can be compared to select commercially available latex polymers, in particular, in terms of elongation of break, to the films from poly (vinyl acetate) latexes, indicating their potential in adhesive applications ${ }^{33}$.

The cross-cut method was employed in order to test the effect of biobased copolymer composition on the adhesion of formulated coatings to the metal substrate. The data indicated that higher HOSBM content in latexes promotes polymer adhesion, making the synthesized biobased latexes relevant in terms of commercial applications ${ }^{34}$.

Table 4. Properties of CBM-HOSBM biobased latex coatings.

\begin{tabular}{|c|c|c|c|c|c|c|}
\hline \multicolumn{2}{|c|}{$\begin{array}{l}\text { Composition in feed, } \\
\% \text { wt. }\end{array}$} & \multirow[t]{2}{*}{$\begin{array}{c}\text { Thickness, } \\
\mu \mathrm{m}\end{array}$} & \multirow[t]{2}{*}{$\begin{array}{c}\text { Pencil } \\
\text { hardness }^{1}\end{array}$} & \multirow[t]{2}{*}{$\begin{array}{c}\text { X-Cut } \\
\text { adhesion }^{2}\end{array}$} & \multirow[t]{2}{*}{$\begin{array}{c}\text { Water } \\
\mathbf{D R}^{3}\end{array}$} & \multirow[t]{2}{*}{$\begin{array}{c}\text { MEK } \\
\text { DR }^{3}\end{array}$} \\
\hline CBM & HOSBM & & & & & \\
\hline 75 & 25 & $97.8 \pm 14$ & $8 \mathrm{H}$ & $2 \mathrm{~B}$ & 256 & 52 \\
\hline 50 & 50 & $62.2 \pm 13$ & $5 \mathrm{H}$ & $3 B$ & 294 & 32 \\
\hline 25 & 75 & $84.0 \pm 23$ & $3 \mathrm{H}$ & $5 \mathrm{~B}$ & 285 & 31 \\
\hline 10 & 90 & $92.7 \pm 24$ & $2 \mathrm{H}$ & $5 B$ & 274 & 29 \\
\hline
\end{tabular}

${ }^{1}$ ASTM D3363-05; ${ }^{2}$ ASTM $3359 ;{ }^{3}$ ASTM D4752.

Beyond the improvement of mechanical properties, the incorporation of both highly hydrophobic fatty acid fragments of HOSBM and CBM into latex copolymers improved the water resistance of the coatings, as water double rubs (water DR) testing demonstrates (Table 4). Using the double rubs technique, solvent (methyl ethyl ketone, MEK) resistance of the biobased latex coatings was also examined. Data showed that MEK resistance increased with increasing crosslink density of the polymer networks. 
For latex polymers synthesized from CBM and HOSBM, the biorenewable carbon index (BCI) was calculated. BCI shows the percentage of carbon atoms which originate from animal or vegetable origins in each macromolecule. It is considered to be a robust parameter in the evaluation of material biorenewability. Based on the final polymer composition, the calculated BCIs of latexes showed similar values for poly (CBM), poly (HOSBM) and poly(CBM-co-HOSBM), varying in the range of $77.5-78.5$. This can be expected based on the similarity between the chemical structures of CBM and HOSBM. Thus, a vast majority of carbon atoms in the synthesized latexes is from natural origin.

\section{Conclusions}

Vinyl monomers derived from cardanol (CBM) and high oleic soybean oil (HOSBM) were successfully (co)polymerized in order to determine their reactivity in radical (co)polymerization, and feasibility in the synthesis of biobased latexes using miniemulsion.

The rate of (co)polymerization depends noticeably on the monomer unsaturation. Due to the allylic termination (pronounced for more unsaturated CBM), chain propagation coexists with effective chain transfer. Nevertheless, most (> 90\%) fatty acid double bonds remain intact and can be used for post-polymerization reactions (cross-linking). $Q$ and $e$ values were similar for CBM and HOSBM, as it can be expected due to similarities in their chemical structures. The obtained results indicated that both monomers are well suited for chain copolymerization.

Miniemulsion (co)polymerization of CBM and HOSBM yielded stable aqueous polymer dispersions with latex particle diameters of $50-130 \mathrm{~nm}$. Thermal analysis showed that the synthesized biobased copolymers possessed lower $T_{g}\left(-35-10^{\circ} \mathrm{C}\right)$ values, which increased with increasing HOSBM content. Tensile testing showed that mechanical properties of crosslinked latex films depend considerably on the polymer network crosslinking density, which can be controlled by varying the ratio of CBM and HOSBM.

Both biobased fragments contributed to the mechanical properties of the resulting polymeric materials. The incorporation of CBM fragments enhanced Young's modulus of the films, whereas the HOSBM constituents made them softer and more flexible. Even small (10 wt. \%) fractions of CBM can provide noticeable strength to the soft latex polymer material based entirely on HOSBM. All crosslinked latex films from HOSBM and CBM demonstrated decent properties and performance in terms of pendulum and pencil hardness, water and solvent resistance, as well as adhesion to the steel substrate. The obtained results clearly demonstrate the potential of vinyl monomers derived from cardanol and high oleic soybean 
oil as candidates for the synthesis of polymeric materials with higher biorenewable carbon index (BCI) through radical copolymerization.

To best of our knowledge, latexes from fully renewable plant-based monomer mixtures have not been reported in literature.

\section{Author Information}

\section{Corresponding Authors}

Andriy Voronov, Coatings and Polymeric Materials Department, North Dakota State University, Dep.2760, PO Box 6050, Fargo, ND, 581-5-6050, Phone: + 1701231 9563, Email: andriy.voronov@ndsu.edu

Sylvain Caillol, Institut Charles Gerhardt Montpellier, 8 Rue Ecole Normale, 34296 Montpellier Cedex 5, France, Phone 04671443 27, E-mail: sylvain.caillol@enscm.fr

\section{Notes}

The authors declare no competing financial interests.

\section{References}

(1)Al. Gandini, Macromolecules, 2008, 41 (24), 9491-9504.

(2) Moreno, M. Goikoetxea, J. C. de la Cal, M. J. Barandiaran, J. Polym. Sci., Part A: Polym. Chem., 2014, 52, 3543-3549.

(3) D.J. Kalita, I. Tarnavchyk, M. Sibi, B. R. Moser, D.C. Webster, B.J. Chisholm Progress in Organic Coatings 2018, 125, 453-462.

(4) Wool, R. P.; Sun, X. S. Bio-Based Polymers and Composites; Elsevier: Amsterdam, 2005.

(5) Y. Zhu, Ch. Romain, Ch. K. Williams Nature 2016, 540, 354-362.

(6) Sharma, V.; Kundu, P. P. Addition Polymers from Natural Oils A Review. Prog. Polym. Sci. 2006, 31, 983-1008.

(7) R.P. Babu , K.O. Connor, R. Seeram Progress in Biomaterials 2013, 2-8.

(8) G. Lligadas, J.C. Ronda, M. Galià, V. Cádiz Materials Today 2013, 16(9), 337-343.

(9) Y. Lu, R. C. Larock Chem. Sus. Chem., 2009, 2, 136 - 147.

(10) J. Lomège, V. Lapinte, C. Negrell, JJ. Robin, S. Caillol, Biomacromolecules, 2019, 20 (1), 4-26.

(11) S. Molina-Gutiérrez, V. Ladmiral, R. Bongiovanni, S. Caillol, P. Lacroix-Desmazes, Green Chemistry, 2019, 21, 36-53. 
(12) K. Kingsley, O. Shevchuk, Z. Demchuk, S. Voronov, A. Voronov Industrial Crops \& Products 109, 2017, 274-280.

(13) E. Kaya, Sh. K. Mendon, D. Delatte, J. W. Rawlins, S. F. Thames Macromol. Symp., 2013, 324, 95-106.

(14) C. Voirin, S. Caillol, N. V. Sadavarte, B. V. Tawade, B. Boutevin, P. P. Wadgaonkar Polymer Chemistry, 2014, 5, 3142-3162.

(15) Z. Demchuk, O. Shevchuk, I. Tarnavchyk, V. Kirianchuk, A. Kohut, S. Voronov, A. Voronov, ACS Sustainable Chem. Eng. 2016, 4, 6974-6980.

(16) Z. Demchuk, A. Kohut, S. Voronov, A. Voronov ACS Sustainable Chem. Eng.,2018, 6, $2780-2786$.

(17) Z. Demchuk, V. Kirianchuk, K. Kingsley, S. Voronov, A. Voronov J. of Theor. and Appl. Nanotech., 2018, 6, 29-37.

(18) A. Greco, Alf. Maffezzoli, Polym. Degradation and Stability, 132, 2016, 213-219.

(19) R. Saladino, V. Neri, En. Mincione, S. Marini, M. Coletta, C. Fiorucci, P. Filippone J. Chem. Soc., Perkin Trans. 1, 2000, 581-586.

(20) André Leandro da Silva et al. Polímeros, 2016, 26(1), 21-29.

(21) B. Briou, S. Caillol, J.J. Robin, V. Lapinte Industrial Crops and Products, 2019, 130, 18.

(22) E. Calo, A. Maffezzoli, G. Mele, F. Martina, S. E. Mazzetto, A. Tarziac, C. Stifanic Green Chem., 2007, 9, 754-759.

(23) M. M. Moreira, L. R. Rocha da Silva, T.A.D. Mendes, S. L. Santiago, S. E. Mazzetto, D. Lomonaco, V. P. Feitosa Dental Materials 2018, 34 (8), 1144-1153.

(24) W.S.J. Li, C. Negrell, V. Ladmiral, J. Lai-Kee-Him, P. Bron, P. Lacroix-Desmazes, C. Joly-Duhamel, S. Caillol Polym. Chem, 2018, 9, 2468-2477.

(25) V. Ladmiral et al. European Polym. J., 2017, 93,785-794.

(26) A. Kohut, Z. Demchuk, K. Kingsley, S. Voronov, A. Voronov European Polym. J., 2018, 108, 322-328.

(27) Iz. M. Barszczewska-Rybarek, A. Korytkowska-Walach, M. Kurcok, G. Chladek, J. Kasperski Acta of Bioengineering and Biomechanics 2017, 19(1),47-53.

(28) Z. Demchuk, O. Shevchuk, I. Tarnavchyk, V. Kirianchuk, M. Lorenson, A. Kohut, S. Voronov, A. Voronov ACS Omega, 2016, 1 (6), 1374-1382.

(29) G. Odian: Principles of Polymerization Ch. 6, 2004, 464-543.

(30) Morrison, R. T., Boyd, R. N. Organic Chemistry, $4^{\text {th }}$ Edition, Allyn and Bacon, Inc., 1983. 
(31) M. Wen, Li V. Ng, J. A. Payne, L. F. Francis, L. E. Scriven, A. V. McCormick $I S \& T^{\prime} s$ 50th Annual Conference, 1997, 564-569.

(32) Pytel, J. Biorenewable carbon index for green formulations HAPPI , 2009, 58-60.

(33) Yildirim H. Erbil Vinyl Acetate Emulsion Polymerization and copolymerization with Acrylic Monomers CRC Press LLC, 2000, 135.

(34) K.A. Shaffei, A.B. Moustafa, A.I. Hamed International Journal of Polymer Science, 2009, 1-6.

\section{TOC graph}

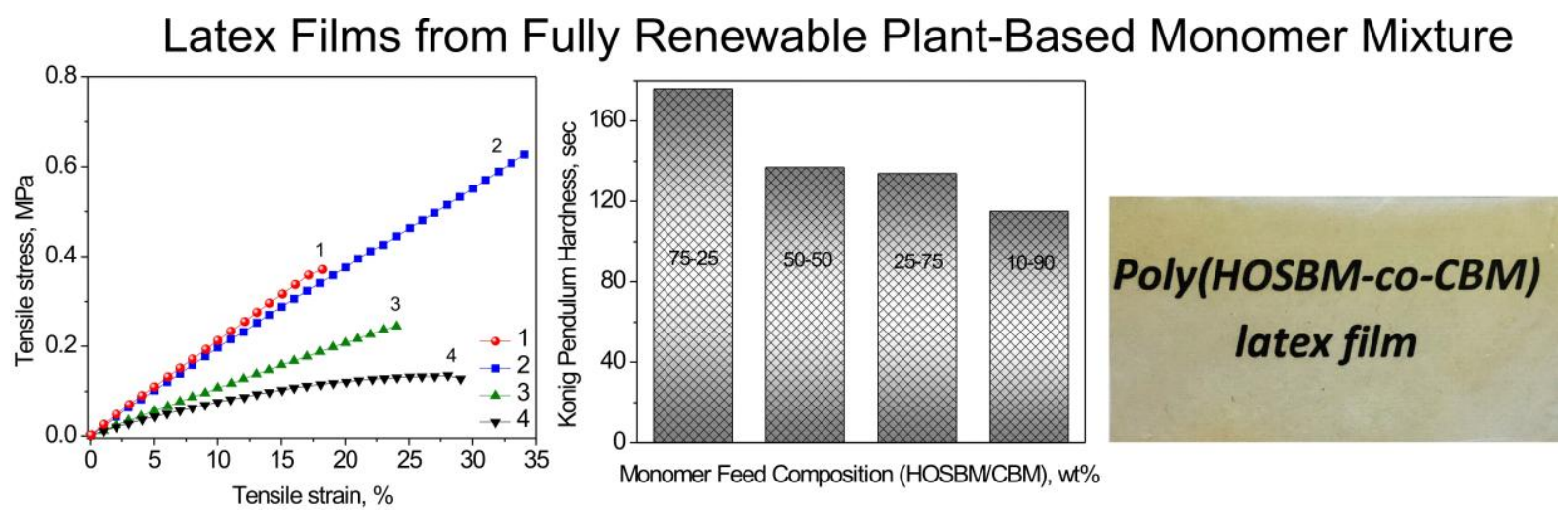

